\title{
Numerical Methods for Semilinear Fractional Diffusion Equations with Time Delay
}

\author{
Shuiping Yang ${ }^{1}$, Yubin Liu ${ }^{1}$, Hongyu Liu ${ }^{2, *}$ and Chao Wang ${ }^{3,4, *}$ \\ 1 School of Mathematics and Big Data Science, Huizhou University, Huizhou, \\ Guangdong 516007, China \\ 2 Department of Mathematics, City University of Hong Kong, Kowloon, Hong Kong \\ ${ }^{3}$ Department of Mathematics, Southern University of Science and Technology, \\ Shenzhen, Guangdong 518055, China \\ ${ }^{4}$ Guangdong Provincial Key Laboratory of Computational Science and Material Design, \\ Southern University of Science and Technology, Shenzhen, Guangdong 518055, China
}

Received 19 December 2020; Accepted (in revised version) 12 February 2021

\begin{abstract}
In this paper, we consider the numerical solutions of the semilinear Riesz space-fractional diffusion equations (RSFDEs) with time delay, which constitute an important class of differential equations of practical significance. We develop a novel implicit alternating direction method that can effectively and efficiently tackle the RSFDEs in both two and three dimensions. The numerical method is proved to be uniquely solvable, stable and convergent with second order accuracy in both space and time. Numerical results are presented to verify the accuracy and efficiency of the proposed numerical scheme.
\end{abstract}

AMS subject classifications: 65N15, 65N30

Key words: Semilinear Riesz space fractional diffusion equations with time delay, implicit alternating direction method, stability and convergence.

\section{Introduction}

It is widely known that mathematical models with time delay are of fundamental importance in many scientific and engineering applications, including economics, physics, population ecology and medicine. As a result, the theoretical analysis and numerical computation of many differential equations with time delay have been studied by numerous researchers $[1,16,23,24]$. Fractional differential equations with delay have received a lot of attentions $[2,3,8,40,43,52,63,64]$ as a result of the development of fractional calculus in

${ }^{*}$ Corresponding author.

Emails: yang52053052@163.com (S. Yang), hongyliu@cityu.edu.hk (H. Liu), wangc3@sustech.edu.cn (C. Wang) 
science and engineering $[5,6,26,27,39,47-49]$. As a typical example, the fractional Bloch equation with delay was proposed to depict the nuclear magnetic resonance [2]. Most of the problems for fractional differential equations and fractional differential equations with delay can not be solved analytically. Thereby, numerical treatment for such type of equations becomes a hot topic in the communities of numerical mathematics. In recent years, the attention on the numerical computations of fractional differential equations has been discussed by many researchers [7,10-14, 19, 20, 22, 42, 51, 54, 55, 57, 60-62]. For example, in [59] the authors considered a class of variable order fractional advection diffusion equation with a nonlinear reaction term. The two-dimensional RSFDEs with nonlinear reaction term was studied in [21]. Recently, the alternating direction implicit Galerkin-Legendre spectral method was proposed to solve the two-dimensional nonlinear reaction-diffusion equations with the Riesz space-fractional derivatives in [53]. In [58], the authors developed the finite element method to solve the two-dimensional nonlinear Riesz space fractional derivatives Fisher' s equation. In [56], a finite difference scheme was proposed for the two-dimensional diffusion equation with the Riesz spacefractional derivatives.

Very recently, some researchers developed methods on numerical solutions of fractional PDEs with time delay. The finite difference method was developed for solving the semi-linear space-fractional diffusion equations with time delay in [15]. In [45], the authors studied a linearized Crank-Nicolson method for solving the nonlinear fractional diffusing equation with multi-delay. In [41], the authors proposed the invariant subspace approach to solve a class of time-fractional partial differential equations with time delay. However, all of the works mentioned above focus on the one dimensional fractional PDEs with delay. In this paper, we shall develop high order schemes for the semilinear Riesz space-fractional diffusion equations with time delay in both two and three dimensions.

The rest of the paper is organized as follows. In Section 2, we present the numerical methods for the two-dimensional and three-dimensional semilinear RSFDEs with time delay. The stability and convergence of the method are proved in Section 3. Finally, we carry out some numerical experiments to confirm the theoretical results of the proposed method in Section 4.

\section{Numerical methods for semilinear RSFDEs with time delay}

In this paper, we consider the following two-dimensional and three-dimensional semilinear RSFDEs with time delay:

$$
\begin{cases}\frac{\partial u(x, y, t)}{\partial t}=K_{x} \frac{\partial^{\alpha} u(x, y, t)}{\partial|x|^{\alpha}}+K_{y} \frac{\partial^{\beta} u(x, y, t)}{\partial|y|^{\beta}}+f(x, y, t, u, u(x, y, t-s)), & 1<\alpha, \beta \leq 2, \quad(x, y, t) \in \Omega \times[0, T], \\ u(x, y, t)=0, & (x, y) \in \partial \Omega, \quad t \in[0, T] \\ u(x, y, t)=\varphi(x, y, t), & (x, y, t) \in \Omega \times[-s, 0],\end{cases}
$$


and

$$
\left\{\begin{aligned}
\frac{\partial u(x, y, z, t)}{\partial t}= & K_{x} \frac{\partial^{\alpha} u(x, y, z, t)}{\partial|x|^{\alpha}}+K_{y} \frac{\partial^{\beta} u(x, y, z, t)}{\partial|y|^{\beta}}+K_{z} \frac{\partial^{\gamma} u(x, y, z, t)}{\partial|z|^{\gamma}} \\
& \quad+g(x, y, z, t, u, u(x, y, z, t-s))
\end{aligned} \quad \begin{array}{rl}
1<\alpha, \beta, \gamma \leq 2, \quad(x, y, z, t) \in\left(0, L_{x}\right) \times\left(0, L_{y}\right) \times\left(0, L_{z}\right) \times[0, T], \\
u(0, y, z, t)=u\left(L_{x}, y, z, t\right)=0, \quad u(x, 0, z, t)=u\left(x, L_{y}, z, t\right)=0 \\
u(x, y, 0, t)=u\left(x, y, L_{z}, t\right)=0, \\
u(x, y, z, t)=\varphi(x, y, z, t), \quad(x, y, z, t) \in\left(0, L_{x}\right) \times\left(0, L_{y}\right) \times\left(0, L_{z}\right) \times[-s, 0]
\end{array}\right.
$$

where $\Omega=\left(0, L_{x}\right) \times\left(0, L_{y}\right)$, and $K_{x}, K_{y}, K_{z} \geq 0$ signify the dispersion coefficients, $s>0$ is a time delay.

Throughout the paper, we assume that

(A1) the solutions of the problem (2.1) and (2.2) have piecewise smooth derivatives with respect to $t$ in the subintervals $(n s,(n+1) s), n=0,1,2, \cdots$,

(A2) the RHS function $f$ in Eq. (2.1) has the first-order continuous partial derivatives with respect to its fourth and fifth arguments. Moreover, the following Lipschitz condition

$$
\left|f\left(x, y, t, u_{1}, u_{2}\right)-f\left(x, y, t, \bar{u}_{1}, \bar{u}_{2}\right)\right| \leq Ł_{1}\left|u_{1}-\bar{u}_{1}\right|+Ł_{2}\left|u_{2}-\bar{u}_{2}\right|
$$

holds for all $u_{1}, u_{2}, \bar{u}_{1}, \bar{u}_{2}$ over $\left[0, L_{x}\right] \times\left[0, L_{y}\right] \times[0, T]$ with the Lipschitz constants $\beta_{1}$, $\beta_{2}$. Similarly, the RHS function $g$ in Eq. (2.2) has the first-order continuous partial derivatives with respect to its fifth and sixth arguments, and the following Lipschitz condition

$$
\left|g\left(x, y, z, t, u_{1}, u_{2}\right)-g\left(x, y, z, t, \bar{u}_{1}, \bar{u}_{2}\right)\right| \leq \bar{L}_{1}\left|u_{1}-\bar{u}_{1}\right|+\bar{L}_{2}\left|u_{2}-\bar{u}_{2}\right|
$$

holds for all $u_{1}, u_{2}, \bar{u}_{1}, \bar{u}_{2}$ over $\left[0, L_{x}\right] \times\left[0, L_{y}\right] \times\left[0, L_{z}\right] \times[0, T]$, where $L_{1}, L_{1}, \bar{L}_{1}, \bar{L}_{2}$ are Lipschitz constants.

The Riesz space fractional operators $\frac{\partial^{\alpha} u}{\partial|x|^{\alpha}}, \frac{\partial^{\beta} u}{\partial|y|^{\beta}}, \frac{\partial^{\gamma} u}{\partial|z|^{\gamma}}$ are defined as (see [20])

$$
\begin{aligned}
\frac{\partial^{\alpha} u}{\partial|x|^{\alpha}} & =-c_{\alpha}\left[{ }_{0} D_{x}^{\alpha} u+{ }_{x} D_{L_{x}}^{\alpha} u\right], \\
\frac{\partial^{\beta} u}{\partial|y|^{\beta}} & =-c_{\beta}\left[{ }_{0} D_{y}^{\beta} u+{ }_{y} D_{L_{y}}^{\beta} u\right], \\
\frac{\partial^{\gamma} u}{\partial|z|^{\gamma}} & =-c_{\gamma}\left[{ }_{0} D_{z}^{\gamma} u+{ }_{z} D_{L_{z}}^{\gamma} u\right],
\end{aligned}
$$

where

$$
c_{\alpha}=\frac{1}{2 \cos \left(\frac{\pi \alpha}{2}\right)}, \quad c_{\beta}=\frac{1}{2 \cos \left(\frac{\pi \beta}{2}\right)}, \quad c_{\gamma}=\frac{1}{2 \cos \left(\frac{\pi \gamma}{2}\right)},
$$


and

$$
\begin{aligned}
& { }_{0} D_{x}^{\alpha} u(x, y, t)=\frac{1}{\Gamma(2-\alpha)} \frac{\partial^{2}}{\partial x^{2}} \int_{0}^{x}(t-\tau)^{1-\alpha} u(\tau, y, t) d \tau, \\
& { }_{x} D_{L_{x}}^{\alpha} u(x, y, t)=\frac{1}{\Gamma(2-\alpha)} \frac{\partial^{2}}{\partial x^{2}} \int_{x}^{L_{x}}(t-\tau)^{1-\alpha} u(\tau, y, t) d \tau .
\end{aligned}
$$

In a similar manner, we can define the other space Riesz fractional derivatives with respect to $y$ and $z$, respectively.

Next, we define $\tau=T / N, t_{n}=n \tau, n=0,1, \cdots, N, s=m \tau$. Let $h_{x}=\frac{L_{x}}{M_{x}}, x_{i}=i h_{x}$ for $i=0,1, \cdots, M_{x}$, and $h_{y}=\frac{L_{y}}{M_{y}}, y_{j}=j h_{y}$ for $j=0,1, \cdots, M_{y}, h_{z}=\frac{L_{z}}{M_{z}}, z_{k}=k h_{z}$ for $k=0,1, \cdots, M_{z}$ be the space step-sizes. Define $u_{i, j}^{n}$ as the numerical approximation to $u\left(x_{i}, y_{j}, t_{n}\right)$ and $u_{i, j, k}^{n}$ as the numerical approximation to $u\left(x_{i}, y_{j}, z_{k}, t_{n}\right)$. Denote $\bar{\Omega}_{h}=\left\{\left(x_{i}, y_{j}\right) \mid 0 \leq i \leq M_{x}, 0 \leq j \leq M_{y}\right\}$, $\Omega_{h}=\bar{\Omega}_{h} \cap \Omega, \partial \Omega_{h}=\bar{\Omega}_{h} \cap \partial \Omega$,

$$
\begin{aligned}
& V_{h}=\left\{v \mid v=\left\{v_{i, j}\right\}, 0 \leq i \leq M_{x}, 0 \leq j \leq M_{y}\right\}, \\
& \stackrel{\circ}{V}_{h}=\left\{v \mid v=\left\{v_{i, j}\right\}, 0 \leq i \leq M_{x}, 0 \leq j \leq M_{y}, v_{i, j}=0, \text { if }\left(x_{i}, y_{j}\right) \in \partial \Omega_{h}\right\} .
\end{aligned}
$$

In addition, we define the following operators

$$
\delta_{t} v_{i, j}^{n+1 / 2}=\frac{1}{\tau}\left(v_{i, j}^{n+1}-v_{i, j}^{n}\right), \quad v_{i, j}^{n+1 / 2}=\frac{1}{2}\left(v_{i, j}^{n+1}+v_{i, j}^{n}\right) .
$$

In the following, we review some useful lemmas.

Lemma 2.1 ([20]). Suppose that $1<\gamma<2, v(x) \in C^{5}[0, L]$. If $v(x)=0, \forall x \in(-\infty, 0] \cup[L,+\infty)$, then

$$
\begin{aligned}
& { }_{0} D_{x}^{\gamma} v\left(x_{i}\right)=\frac{1}{h^{\gamma}} \sum_{k=0}^{i+1} \omega_{\gamma}^{(k)} v\left(x_{i-k+1}\right)+\mathcal{O}\left(h^{2}\right), \\
& { }_{x} D_{L}^{\gamma} v\left(x_{i}\right)=\frac{1}{h^{\gamma}} \sum_{k=0}^{m-i+1} \omega_{\gamma}^{(k)} v\left(x_{i+k-1}\right)+\mathcal{O}\left(h^{2}\right),
\end{aligned}
$$

where

$$
\begin{aligned}
& \omega_{\gamma}^{(0)}=\frac{\gamma}{2} g_{0}^{(\gamma)}, \\
& \omega_{\gamma}^{(k)}=\frac{\gamma}{2} g_{k}^{(\gamma)}+\frac{2-\gamma}{2} g_{k-1}^{(\gamma)}, \\
& g_{0}^{(\gamma)}=1, g_{k}^{(\gamma)}=\left(1-\frac{\gamma+1}{k}\right) g_{k-1}^{(\gamma)}, \quad k=1,2, \cdots .
\end{aligned}
$$

Lemma 2.2 ([20]). Suppose that $1<\gamma \leq 2$, then $\left\{g_{k}^{(\gamma)}\right\}$ satisfy

$$
\left\{\begin{array}{l}
g_{0}^{(\gamma)}=1, \quad g_{1}^{(\gamma)}=-\gamma, \quad g_{2}^{(\gamma)}=\frac{\gamma(\gamma-1)}{2}>0 \\
1 \geq g_{2}^{(\gamma)} \geq g_{3}^{(\gamma)} \geq \cdots \geq 0, \quad \sum_{k=0}^{\infty} g_{k}^{(\gamma)}=0, \quad \sum_{k=0}^{m} g_{k}^{(\gamma)}<0, \quad m \geq 1 .
\end{array}\right.
$$


Lemma 2.3 ([20]). Suppose that $1<\gamma \leq 2$, then $\left\{\omega_{\gamma}^{(k)}\right\}$ satisfy

$$
\left\{\begin{array}{l}
\omega_{\gamma}^{(0)}=\frac{\gamma}{2}, \quad \omega_{\gamma}^{(1)}=\frac{2-\gamma-\gamma^{2}}{2}<0, \quad \omega_{\gamma}^{(2)}=\frac{\gamma\left(\gamma^{2}+\gamma-4\right)}{4} \\
1 \geq \omega_{\gamma}^{(2)} \geq \omega_{\gamma}^{(3)} \geq \cdots \geq 0, \quad \sum_{k=0}^{\infty} \omega_{\gamma}^{(k)}=0, \quad \sum_{k=0}^{m} \omega_{\gamma}^{(k)}<0, \quad m \geq 2
\end{array}\right.
$$

\subsection{The two-dimensional case}

Now, in order to approximate (2.1), we use

$$
\left.\frac{\partial u(x, y, t)}{\partial t}\right|_{\left(x_{i}, y_{j}, t_{n+\frac{1}{2}}\right)}=\frac{u_{i, j}^{n+1}-u_{i, j}^{n}}{\tau}+\mathcal{O}\left(\tau^{2}\right)=\delta_{t} u_{i, j}^{n+1 / 2}+\mathcal{O}\left(\tau^{2}\right) .
$$

According to the method of [20], we have

$$
\begin{aligned}
& \left.\frac{\partial^{\alpha} u(x, y, t)}{\partial|x|^{\alpha}}\right|_{\left(x_{i}, y_{j}, t_{n+\frac{1}{2}}\right)} \\
& =-\frac{c_{\alpha}}{2\left(h_{x}\right)^{\alpha}}\left[\left(\sum_{l=0}^{i+1} \omega_{\alpha}^{(l)} u\left(x_{i-l+1}, y_{j}, t_{n+1}\right)+\sum_{l=0}^{M_{x}-i+1} \omega_{\alpha}^{(l)} u\left(x_{i+l-1}, y_{j}, t_{n+1}\right)\right)\right. \\
& \left.+\left(\sum_{l=0}^{i+1} \omega_{\alpha}^{(l)} u\left(x_{i-l+1}, y_{j}, t_{n}\right)+\sum_{l=0}^{M_{x}-i+1} \omega_{\alpha}^{(l)} u\left(x_{i+l-1}, y_{j}, t_{n}\right)\right)\right]+\mathcal{O}\left(h_{x}^{2}\right), \\
& \left.\frac{\partial^{\beta} u(x, y, t)}{\partial|y|^{\beta}}\right|_{\left(x_{i}, y_{j}, t_{n+\frac{1}{2}}\right)} \\
& =-\frac{c_{\beta}}{2\left(h_{y}\right)^{\beta}}\left[\left(\sum_{l=0}^{j+1} \omega_{\beta}^{(l)} u\left(x_{i}, y_{j-l+1}, t_{n+1}\right)+\sum_{l=0}^{M_{y}-j+1} \omega_{\beta}^{(l)} u\left(x_{i}, y_{j+l-1}, t_{n+1}\right)\right)\right. \\
& \left.+\left(\sum_{l=0}^{j+1} \omega_{\beta}^{(l)} u\left(x_{i}, y_{j-l+1}, t_{n}\right)+\sum_{l=0}^{M_{y}-j+1} \omega_{\beta}^{(l)} u\left(x_{i}, y_{j+l-1}, t_{n}\right)\right)\right]+\mathcal{O}\left(h_{y}^{2}\right) .
\end{aligned}
$$

For the nonlinear reaction term, by using the Taylor expansion, we can obtain the following approximation:

$$
\begin{aligned}
& f\left(x_{i}, y_{j}, t_{n+\frac{1}{2}}, u\left(x_{i}, y_{j}, t_{n+\frac{1}{2}}\right), u\left(x_{i}, y_{j}, t_{n+\frac{1}{2}}-s\right)\right) \\
= & f\left(x_{i}, y_{j}, t_{n+\frac{1}{2}}, \frac{3}{2} u\left(x_{i}, y_{j}, t_{n}\right)-\frac{1}{2} u\left(x_{i}, y_{j}, t_{n-1}\right), u\left(x_{i}, y_{j}, t_{n+\frac{1}{2}}-s\right)\right)+\mathcal{O}\left(\tau^{2}\right) \\
\sim & f\left(x_{i}, y_{j}, t_{n+\frac{1}{2}}, \frac{3}{2} u_{i, j}^{n}-\frac{1}{2} u_{i, j}^{n-1}, u_{i, j}^{n+\frac{1}{2}-m}\right)+\mathcal{O}\left(\tau^{2}\right) .
\end{aligned}
$$


Therefore, the numerical method for (2.1) is determined by the following finite difference equation

$$
\begin{aligned}
\frac{u_{i, j}^{n}-u_{i, j}^{n-1}}{\tau}=- & \frac{K_{x} c_{\alpha}}{2\left(h_{x}\right)^{\alpha}}\left[\left(\sum_{l=0}^{i+1} \omega_{\alpha}^{(l)} u\left(x_{i-l+1}, y_{j}, t_{n+1}\right)+\sum_{l=0}^{M_{x}-i+1} \omega_{\alpha}^{(l)} u\left(x_{i+l-1}, y_{j}, t_{n+1}\right)\right)\right. \\
& \left.\cdot\left(\sum_{l=0}^{i+1} \omega_{\alpha}^{(l)} u\left(x_{i-l+1}, y_{j}, t_{n}\right)+\sum_{l=0}^{M_{x}-i+1} \omega_{\alpha}^{(l)} u\left(x_{i+l-1}, y_{j}, t_{n}\right)\right)\right] \\
& -\frac{K_{y} c_{\beta}}{2\left(h_{y}\right)^{\beta}}\left[\left(\sum_{l=0}^{j+1} \omega_{\beta}^{(l)} u\left(x_{i}, y_{j-l+1}, t_{n+1}\right)+\sum_{l=0}^{M_{y}-j+1} \omega_{\beta}^{(l)} u\left(x_{i}, y_{j+l-1}, t_{n+1}\right)\right)\right. \\
& \left.\cdot\left(\sum_{l=0}^{j+1} \omega_{\beta}^{(l)} u\left(x_{i}, y_{j-l+1}, t_{n}\right)+\sum_{l=0}^{M_{y}-j+1} \omega_{\beta}^{(l)} u\left(x_{i}, y_{j+l-1}, t_{n}\right)\right)\right] \\
& +f\left(x_{i}, y_{j}, t_{n+\frac{1}{2}}, \frac{3}{2} u_{i, j}^{n}-\frac{1}{2} u_{i, j}^{n-1}, u_{i, j}^{n+\frac{1}{2}-m}\right) .
\end{aligned}
$$

Clearly, the numerical method (2.12) is consistent with order $\mathcal{O}\left(\tau^{2}+h_{x}^{2}+h_{y}^{2}\right)$.

Define the following fractional partial difference operators:

$$
\begin{aligned}
\delta_{x}^{\alpha} u_{i, j}^{n} & =-\frac{K_{x} c_{\alpha}}{\left(h_{x}\right)^{\alpha}}\left[\sum_{l=0}^{i+1} \omega_{\alpha}^{(l)} u\left(x_{i-l+1}, y_{j}, t_{n}\right)+\sum_{l=0}^{M_{x}-i+1} \omega_{\alpha}^{(l)} u\left(x_{i+l-1}, y_{j}, t_{n}\right)\right], \\
\delta_{y}^{\beta} u_{i, j}^{n} & =-\frac{K_{y} c_{\beta}}{\left(h_{y}\right)^{\beta}}\left[\sum_{l=0}^{j+1} \omega_{\beta}^{(l)} u\left(x_{i}, y_{j-l+1}, t_{n}\right)+\sum_{l=0}^{M_{y}-j+1} \omega_{\beta}^{(l)} u\left(x_{i}, y_{j+l-1}, t_{n}\right)\right] .
\end{aligned}
$$

By means of these operator definitions, the numerical method (2.12) can be written as

$$
\delta_{t} u_{i, j}^{n+1 / 2}=\delta_{x}^{\alpha} u_{i, j}^{n+1 / 2}+\delta_{y}^{\beta} u_{i, j}^{n+1 / 2}+f\left(x_{i}, y_{j}, t_{n+\frac{1}{2}}, \frac{3}{2} u_{i, j}^{n}-\frac{1}{2} u_{i, j}^{n-1}, u_{i, j}^{n+\frac{1}{2}-m}\right)
$$

or

$$
\begin{aligned}
& \left(1-\frac{\tau}{2} \delta_{x}^{\alpha}-\frac{\tau}{2} \delta_{y}^{\beta}\right) u_{i, j}^{n+1} \\
= & \left(1+\frac{\tau}{2} \delta_{x}^{\alpha}+\frac{\tau}{2} \delta_{y}^{\beta}\right) u_{i, j}^{n}+\tau f\left(x_{i}, y_{j}, t_{n+\frac{1}{2}}, \frac{3}{2} u_{i, j}^{n}-\frac{1}{2} u_{i, j}^{n-1}, u_{i, j}^{n+\frac{1}{2}-m}\right), \\
& 1 \leq i \leq M_{x}-1, \quad 1 \leq j \leq M_{y}-1 .
\end{aligned}
$$

The boundary and initial conditions are discretized as

$$
\begin{array}{ll}
u_{0, j}^{n}=u\left(0, y_{j}, t_{n}\right)=0, & u_{M_{x}, j}^{n}=u\left(L_{x}, y_{j}, t_{n}\right)=0, \\
u_{i, 0}^{n}=u\left(x_{i}, 0, t_{n}\right)=0, & u_{i, M_{y}}^{n}=u\left(x_{i}, L_{y}, t_{n}\right)=0, \\
u_{i, j}^{k}=\varphi\left(i h_{x}, j h_{y}, k \tau\right), & k \leq 0 .
\end{array}
$$


The operator form (2.15) can be written in the following directional separation product form named as alternating direction implicit methods (ADIM)

$$
\begin{aligned}
& \left(1-\frac{\tau}{2} \delta_{x}^{\alpha}\right)\left(1-\frac{\tau}{2} \delta_{y}^{\beta}\right) u_{i, j}^{n+1} \\
= & \left(1+\frac{\tau}{2} \delta_{x}^{\alpha}\right)\left(1+\frac{\tau}{2} \delta_{y}^{\beta}\right) u_{i, j}^{n}+\tau f\left(x_{i}, y_{j}, t_{n+\frac{1}{2}}, \frac{3}{2} u_{i, j}^{n}-\frac{1}{2} u_{i, j}^{n-1}, u_{i, j}^{n+\frac{1}{2}-m}\right), \\
& 1 \leq i \leq M_{x}-1, \quad 1 \leq j \leq M_{y}-1,
\end{aligned}
$$

which introduces an additional perturbation term $C\left[\delta_{x}^{\alpha} \delta_{y}^{\beta}\right]\left(u_{i, j}^{n+1}+u_{i, j}^{n}\right)$. Clearly, this method is consistent with order $\mathcal{O}\left(\tau^{2}+h_{x}^{2}+h_{y}^{2}\right)$.

The method (2.16) can now be solved by the following iterative scheme:

- First, for each fixed $y_{j}$, solve the problem in the $x$-direction to obtain an intermediate solution $\bar{u}_{i, j}^{n}$ in the form

$$
\begin{aligned}
& \left(1-\frac{\tau}{2} \delta_{x}^{\alpha}\right) \bar{u}_{i, j}^{n+1} \\
= & \left(1+\frac{\tau}{2} \delta_{x}^{\alpha}\right)\left(1+\frac{\tau}{2} \delta_{y}^{\beta}\right) u_{i, j}^{n}+\tau f\left(x_{i}, y_{j}, t_{n+\frac{1}{2}}, \frac{3}{2} u_{i, j}^{n}-\frac{1}{2} u_{i, j}^{n-1}, u_{i, j}^{n+\frac{1}{2}-m}\right) .
\end{aligned}
$$

- Second, for each fixed $x_{i}$, solve the problem in the $y$-direction,

$$
\left(1-\frac{\tau}{2} \delta_{y}^{\beta}\right) u_{i, j}^{n+1}=\bar{u}_{i, j}^{n+1}
$$

The boundary and initial conditions are discretized as

$$
\begin{array}{ll}
u_{0, j}^{n}=u\left(0, y_{j}, t_{n}\right)=0, & u_{M_{x}, j}^{n}=u\left(L_{x}, y_{j}, t_{n}\right)=0, \\
u_{i, 0}^{n}=u\left(x_{i}, 0, t_{n}\right)=0, & u_{i, M_{y}}^{n}=u\left(x_{i}, L_{y}, t_{n}\right)=0, \\
\bar{u}_{0, j}=0, & \bar{u}_{M_{x}, j}=0,
\end{array}
$$

and

$$
u_{i, j}^{k}=\varphi\left(i h_{x}, j h_{y}, k \tau\right), \quad k \leq 0
$$

Let

$$
\begin{array}{ll}
r_{x}=\tau \frac{K_{x} c_{\alpha}}{2\left(h_{x}\right)^{\alpha}}, & r_{y}=\tau \frac{K_{y} c_{\beta}}{2\left(h_{y}\right)^{\beta},} \\
D_{x}=\left(d_{i, j}^{x}\right)_{\left(M_{x}-1\right) \times\left(M_{x}-1\right),} & D_{y}=\left(d_{i, j}^{y}\right)_{\left(M_{y}-1\right) \times\left(M_{y}-1\right),}
\end{array}
$$


where

$$
\begin{aligned}
& d_{i, j}^{x}= \begin{cases}r_{x} \omega_{\alpha}^{(i-j+1)} & \text { for } j<i-1, \\
r_{x}\left(\omega_{\alpha}^{(0)}+\omega_{\alpha}^{(2)}\right) & \text { for } j=i-1, \\
2 r_{x} \omega_{\alpha}^{(1)} & \text { for } j=i, \\
r_{x}\left(\omega_{\alpha}^{(0)}+\omega_{\alpha}^{(2)}\right) & \text { for } j=i+1, \\
r_{x} \omega_{\alpha}^{(j-i+1)} & \text { for } j>i+1,\end{cases} \\
& d_{i, j}^{y}= \begin{cases}r_{y} \omega_{\beta}^{(i-j+1)} & \text { for } j<i-1, \\
r_{y}\left(\omega_{\beta}^{(0)}+\omega_{\beta}^{(2)}\right) & \text { for } j=i-1, \\
2 r_{y} \omega_{\beta}^{(1)} & \text { for } j=i, \\
r_{y}\left(\omega_{\beta}^{(0)}+\omega_{\beta}^{(2)}\right) & \text { for } j=i+1, \\
r_{y} \omega_{\beta}^{(j-i+1)} & \text { for } j>i+1 .\end{cases}
\end{aligned}
$$

Let

$$
\left(I-D_{y}\right) u_{v}^{n}=\tilde{u}_{v}, \quad 1 \leq v \leq M_{x}-1,
$$

where $u_{v}^{n}=\left(u_{v, 1}^{n}, u_{v, 2}^{n}, \cdots, u_{v, M_{y}-1}^{n}\right)^{T}, \tilde{u}_{v}=\left(\tilde{u}_{v, 1}^{n}, \tilde{u}_{v, 2}^{n}, \cdots, \tilde{u}_{v, M_{y}-1}^{n}\right)^{T}$. Then (2.17) can be rewritten in the matrix form

$$
\left(I+D_{x}\right) \bar{u}_{w}^{n}=\left(I-D_{x}\right) \tilde{u}_{w}^{*}+\tau f, \quad 1 \leq w \leq M_{y}-1,
$$

where $u_{w}^{*}=\left(\tilde{u}_{1, w}, \tilde{u}_{2, w}, \cdots, \tilde{u}_{M_{x}-1, w}\right)^{T}, \bar{u}_{w}^{n}=\left(\bar{u}_{1, w}^{n}, \bar{u}_{2, w}^{n}, \cdots, \bar{u}_{M_{x}-1, w}^{n}\right)^{T}$,

$$
\begin{gathered}
f=\left(f\left(x_{1}, y_{w}, t_{n+\frac{1}{2}}, \frac{3}{2} u_{1, w}^{n}-\frac{1}{2} u_{1, w}^{n-1}, u_{1, w}^{n+\frac{1}{2}-m}\right), f\left(x_{2}, y_{w}, t_{n+\frac{1}{2}}, \frac{3}{2} u_{2, w}^{n}-\frac{1}{2} u_{2, w}^{n-1}, u_{2, w}^{n+\frac{1}{2}-m}\right),\right. \\
\left.\quad \ldots, f\left(x_{M_{x}-1}, y_{w}, t_{n+\frac{1}{2}}, \frac{3}{2} u_{M_{x}-1, w}^{n}-\frac{1}{2} u_{M_{x}-1, w}^{n-1}, u_{M_{x}-1, w}^{n+\frac{1}{2}-m}\right)\right)^{T} .
\end{gathered}
$$

Similarly, (2.18) can be written in the matrix form

$$
\left(I+D_{y}\right) \hat{u}_{v}^{n+1}=\hat{u}_{v}^{*}, 1 \leq v \leq M_{x}-1,
$$

where $\hat{u}_{v}^{n+1}=\left(u_{v, 1}^{n+1}, u_{v, 2}^{n+1}, \cdots, u_{v, M_{y}-1}^{n+1}\right)^{T}, \hat{u}_{v}^{*}=\left(\bar{u}_{v, 1}^{n}, \bar{u}_{v, 2}^{n}, \cdots, \bar{u}_{v, M_{y}-1}^{n}\right)^{T}$.

\subsection{The three-dimensional case}

Next, we discuss the numerical method for three-dimensional case. We can construct the numerical method for (2.2) as follows:

$$
\begin{aligned}
\delta_{t} u_{i, j, k}^{n+1 / 2}=\delta_{x}^{\alpha} u_{i, j, k}^{n+1 / 2}+\delta_{y}^{\beta} u_{i, j, k}^{n+1 / 2}+\delta_{z}^{\gamma} u_{i, j, k}^{n+1 / 2} & \\
& +g\left(x_{i}, y_{j}, z_{k}, t_{n+\frac{1}{2}}, \frac{3}{2} u_{i, j, k}^{n}-\frac{1}{2} u_{i, j, k}^{n-1}, u_{i, j, k}^{n+\frac{1}{2}-m}\right),
\end{aligned}
$$


or

$$
\begin{aligned}
& \left(1-\frac{\tau}{2} \delta_{x}^{\alpha}-\frac{\tau}{2} \delta_{y}^{\beta}-\frac{\tau}{2} \delta_{z}^{\gamma}\right) u_{i, j, k}^{n+1} \\
= & \left(1+\frac{\tau}{2} \delta_{x}^{\alpha}+\frac{\tau}{2} \delta_{y}^{\beta}+\frac{\tau}{2} \delta_{z}^{\gamma}\right) u_{i, j, k}^{n}+\tau g\left(x_{i}, y_{j}, z_{k}, t_{n+\frac{1}{2},} \frac{3}{2} u_{i, j, k}^{n}-\frac{1}{2} u_{i, j, k}^{n-1}, u_{i, j, k}^{n+\frac{1}{2}-m}\right), \\
& 1 \leq i \leq M_{x}-1, \quad 1 \leq j \leq M_{y}-1, \quad 1 \leq k \leq M_{z}-1,
\end{aligned}
$$

where

$$
\begin{aligned}
& \delta_{x}^{\alpha} u_{i, j, k}^{n}=-\frac{K_{x} c_{\alpha}}{\left(h_{x}\right)^{\alpha}}\left[\sum_{l=0}^{i+1} \omega_{\alpha}^{(l)} u_{i-l+1, j, k}^{n}+\sum_{l=0}^{M_{x}-i+1} \omega_{\alpha}^{(l)} u_{i+l-1, j, k}^{n}\right], \\
& \delta_{y}^{\beta} u_{i, j, k}^{n}=-\frac{K_{y} c_{\beta}}{\left(h_{y}\right)^{\beta}}\left[\sum_{l=0}^{j+1} \omega_{\beta}^{(l)} u_{i, j-l+1, k}^{n}+\sum_{l=0}^{M_{y}-j+1} \omega_{\beta}^{(l)} u_{i, j+l-1, k}^{n}\right], \\
& \delta_{z}^{\gamma} u_{i, j, k}^{n}=-\frac{K_{z} c_{\gamma}}{\left(h_{z}\right)^{\gamma}}\left[\sum_{l=0}^{k+1} \omega_{\gamma}^{(l)} u_{i, j, k-l+1}^{n}+\sum_{l=0}^{M_{z}-k+1} \omega_{\gamma}^{(l)} u_{i, j, k+l-1}^{n}\right] .
\end{aligned}
$$

Moreover, the operator form (2.24) can be written in the following directional separation product form

$$
\begin{aligned}
& \left(1-\frac{\tau}{2} \delta_{x}^{\alpha}\right)\left(1-\frac{\tau}{2} \delta_{y}^{\beta}\right)\left(1-\frac{\tau}{2} \delta_{z}^{\gamma}\right) u_{i, j, k}^{n+1} \\
= & \left(1+\frac{\tau}{2} \delta_{x}^{\alpha}\right)\left(1+\frac{\tau}{2} \delta_{y}^{\beta}\right)\left(1+\frac{\tau}{2} \delta_{z}^{\gamma}\right) u_{i, j, k}^{n}+\tau g\left(x_{i}, y_{j}, z_{k}, t_{n+\frac{1}{2},} \frac{3}{2} u_{i, j, k}^{n}-\frac{1}{2} u_{i, j, k}^{n-1}, u_{i, j, k}^{n+\frac{1}{2}-m}\right),
\end{aligned}
$$

which introduces an additional perturbation error equal to $\mathcal{O}\left(\tau^{2}\right)$. The additional perturbational error is not large compared to the approximation errors for the other terms in (2.24), and this method is consistent with order $\mathcal{O}\left(\tau^{2}+h_{x}^{2}+h_{y}^{2}+h_{z}^{2}\right)$. The method (2.26) can now be solved by the following iterative scheme:

- First, for each fixed $y_{j}, z_{k}$, solve the problem in the $x$-direction to obtain an intermediate solution $\bar{u}_{i, j, k}^{n}$ in the form

$$
\begin{aligned}
\left(1-\frac{\tau}{2} \delta_{x}\right) \bar{u}_{i, j, k}^{n}=(1 & \left.+\frac{\tau}{2} \delta_{x}^{\alpha}\right)\left(1+\frac{\tau}{2} \delta_{y}^{\beta}\right)\left(1+\frac{\tau}{2} \delta_{z}^{\gamma}\right) u_{i, j, k}^{n} \\
& +\tau g\left(x_{i}, y_{j}, z_{k}, t_{n+\frac{1}{2}}, \frac{3}{2} u_{i, j, k}^{n}-\frac{1}{2} u_{i, j, k}^{n-1}, u_{i, j, k}^{n+\frac{1}{2}-m}\right) .
\end{aligned}
$$

- Second, for each fixed $x_{i}, z_{k}$, solve the problem in the $y$-direction,

$$
\left(1-\tau \delta_{y}^{\beta}\right) \hat{u}_{i, j, k}^{n}=\bar{u}_{i, j, k}^{n}
$$

- Finally, for each fixed $x_{i}, y_{j}$, solve the problem in the $z$-direction,

$$
\left(1-\tau \delta_{z}^{\gamma}\right) u_{i, j, k}^{n+1}=\hat{u}_{i, j, k}^{n}
$$


The boundary and initial conditions are discretized as

$$
\begin{array}{ll}
u_{0, j, k}^{n}=u\left(0, y_{j}, z_{k}, t_{n}\right)=0, & u_{M_{x}, j, k}^{n}=u\left(L_{x}, y_{j}, z_{k}, t_{n}\right)=0, \\
u_{i, 0, k}^{n}=u\left(x_{i}, 0, z_{k}, t_{n}\right)=0, & u_{i, M_{y}, k}^{n}=u\left(x_{i}, L_{y}, z_{k}, t_{n}\right)=0, \\
u_{i, j, 0}^{n}=u\left(x_{i}, y_{j}, 0, t_{n}\right)=0, & u_{i, j, L_{z}}^{n}=u\left(x_{i}, y_{j}, L_{z}, t_{n}\right)=0, \\
u_{i, j, k}^{l}=\varphi\left(i h_{x}, j h_{y}, k h_{z}, l \tau\right), & l \leq 0,
\end{array}
$$

and

$$
\begin{array}{ll}
\bar{u}_{0, j, k}^{n}=0, & \bar{u}_{M_{x}, j, k}^{n}=0, \\
\hat{u}_{i, 0, k}^{n}=0, & \hat{u}_{i, M_{y}, k}^{n}=0 .
\end{array}
$$

\section{Theoretical analysis}

Without loss of generality, we present the proofs of stability and convergence of the proposed method for solving the two dimensional semilinear Riesz space fractional diffusion equations with time delay in this paper. The three dimensional case can be proved by following similar arguments. For any grid functions $u, v \in \stackrel{\circ}{h}_{h}$, we define the inner product and norm as

$$
(u, v)=h_{x} h_{y} \sum_{i=1}^{M_{x}-1} \sum_{j=1}^{M_{y}-1} u_{i, j} v_{i, j}, \quad\|u\|=\sqrt{(u, u)} .
$$

In addition, we introduce the following useful lemmas which play important roles in the subsequent analysis.

Lemma 3.1. Suppose that $1<\alpha, \beta \leq 2, D_{x}, D_{y}$ are defined as (2.19a) and (2.19b) respectively. Then $D_{x}, D_{y}$ are strictly diagonally dominant.

Proof. Firstly, we prove that $D_{x}$ is strictly diagonally dominant. Since $1<\alpha \leq 2$ and $K_{x}>0$, so $c_{\alpha}<0$ and $r_{x}<0$. Then $d_{i, i}^{x}>0, d_{i, j}^{x}<0,(i \neq j)$. From Lemma 2.3, we have

$$
\sum_{j=1, j \neq i}^{M_{x}-1}\left|d_{i, j}^{x}\right|=-r_{x}\left(\sum_{j=0, j \neq 1}^{i} \omega_{\alpha}^{(j)}+\sum_{j=0, j \neq 1}^{M_{x}-i} \omega_{\alpha}^{(j)}\right)<-r_{x}\left(-\omega_{\alpha}^{(1)}-\omega_{\alpha}^{(1)}\right)=2 r_{x} \omega_{\alpha}^{(1)}=d_{i, i}^{x}
$$

Then, $D_{x}$ is strictly diagonally dominant. Similarly, we can prove that $D_{y}$ is strictly diagonally dominant.

Lemma 3.2. Suppose that $1<\alpha, \beta \leq 2, D_{x}, D_{y}$ are defined as (2.19a) and (2.19b), respectively. Then $D_{x}, D_{y}$ are symmetric positive definite. 
Proof. In view of (2.19a) and (2.19b), the symmetry of $D_{x}, D_{y}$ is evident. Let $\lambda_{x}$ be one eigenvalue of $D_{x}$. According to the Gerschgorin's circle theorem [46], we have

$$
\left|\lambda_{x}-d_{i, i}^{x}\right| \leq \sum_{j=1, j \neq i}^{M_{x}-1}\left|d_{i, j}^{x}\right| .
$$

Then

$$
d_{i, i}^{x}-\sum_{j=1, j \neq i}^{M_{x}-1}\left|d_{i, j}^{x}\right| \leq \lambda_{x} \leq d_{i, i}^{x}+\sum_{j=1, j \neq i}^{M_{x}-1}\left|d_{i, j}^{x}\right| .
$$

By using Lemma 3.1, we have $\lambda_{x}>0$, thus $D_{x}$ is positive definite. Similarly, we can prove that $D_{y}$ is positive definite.

Lemma 3.3 ([50,56]). For any $u \in \stackrel{\circ}{h}_{h}$, there hold $\left(\delta_{x}^{\alpha} u, u\right) \leq 0$, and $\left(\delta_{y}^{\beta} u, u\right) \leq 0$.

Lemma 3.4 (Gronwall's inequality [44]). Suppose that $\left\{k_{n}\right\}$ and $\left\{p_{n}\right\}$ are nonnegative sequences, and the sequence $\left\{\phi_{n}\right\}$ satisfies

$$
\phi_{0} \leq q_{0}, \quad \phi_{n} \leq q_{0}+\sum_{l=0}^{n-1} p_{l}+\sum_{l=0}^{n-1} p_{l} \phi_{l}, \quad n \geq 1,
$$

where $q_{0} \geq 0$. Then it holds that

$$
\left\{\phi_{n}\right\} \leq\left(q_{0}+\sum_{l=0}^{n-1} p_{l}\right) \exp \left(\sum_{l=0}^{n-1} k_{l}\right), \quad n \geq 1
$$

Theorem 3.1. The difference scheme (2.15) is uniquely solvable.

Proof. Consider the homogeneous form of (2.15) and taking the inner product with $u_{i, j}^{n+1}$, we have

$$
\left(u^{k+1}, u^{k+1}\right)-\frac{\tau}{2}\left(\delta_{x}^{\alpha} u^{k+1}, u^{k+1}\right)-\frac{\tau}{2}\left(\delta_{y}^{\beta} u^{k+1}, u^{k+1}\right)=0 .
$$

It follows from Lemma 3.3 that

$$
\left(u^{k+1}, u^{k+1}\right)=\left\|u^{k+1}\right\|^{2} \leq 0 .
$$

Thus, $\left\|u^{k+1}\right\|=0$ and $u_{i, j}^{k+1}$ can be solved uniquely. The proof is completed.

Theorem 3.2. The difference scheme (2.17)-(2.18) is uniquely solvable.

Proof. According to Lemma 3.2, we know that $I+D_{x}, I+D_{y}$ are strictly diagonally dominant. Then $I+D_{x}, I+D_{y}$ are invertible respectively, which means that the difference scheme (2.17)-(2.18) is uniquely solvable. 
Theorem 3.3. If the solution of the problem (2.1) satisfy the conditions (A1) and (A2), the method (2.15) is convergent. Moreover,

$$
\left\|\epsilon^{n}\right\| \leq C\left(h_{x}^{2}+h_{y}^{2}+\tau^{2}\right), \quad 1 \leq n \leq N,
$$

where $\epsilon_{i, j}^{n}=u\left(x_{i}, y_{j}, t_{n}\right)-u_{i, j}^{n}, 0 \leq i \leq M_{x}, 0 \leq j \leq M_{y}$ denotes the corresponding error, $u_{i, j}^{n},(0 \leq$ $\left.i \leq M_{x}, 0 \leq j \leq M_{y}\right)$ be the numerical solution of the corresponding difference scheme (2.15), $C=c_{1} T \sqrt{L_{x} L_{y}} \exp \left(8 T\left(L_{1}+L_{2}\right)\right), c_{1}$ is a positive constant.

Proof. Obviously, we have

$$
\begin{aligned}
\delta_{t} \epsilon_{i, j}^{n+1 / 2}=\frac{1}{2} \delta_{x}^{\alpha}\left(\epsilon_{i, j}^{n+1}+\epsilon_{i, j}^{n}\right)+\frac{1}{2} \delta_{y}^{\beta}\left(\epsilon_{i, j}^{n+1}+\epsilon_{i, j}^{n}\right)+f\left(x_{i}, y_{j}, t_{n+\frac{1}{2}}, \frac{3}{2} u_{i, j}^{n}-\frac{1}{2} u_{i, j}^{n-1}, u_{i, j}^{n+\frac{1}{2}-m}\right) \\
\quad-f\left(x_{i}, y_{j}, t_{n+\frac{1}{2}}, \frac{3}{2} u\left(x_{i}, y_{j}, t_{n}\right)-\frac{1}{2} u\left(x_{i}, y_{j}, t_{n-1}\right), \frac{1}{2} u\left(x_{i}, y_{j}, t_{n+1-m}\right)\right. \\
\left.+u\left(x_{i}, y_{j}, t_{n-m}\right)\right)+R_{i, j}^{n+1 / 2} .
\end{aligned}
$$

According to (2.12)-(2.15), there exists a positive constant $c_{1}$ such that

$$
\left|R_{i, j}^{n+1 / 2}\right| \leq c_{1}\left(h_{x}^{2}+h_{y}^{2}+\tau^{2}\right), \quad 1 \leq i \leq M_{x}-1, \quad 1 \leq j \leq M_{y}-1, \quad 0 \leq i \leq N-1 .
$$

As a result, we can show that

$$
\delta_{t} \epsilon^{n+1 / 2}=\frac{1}{2} \delta_{x}^{\alpha}\left(\epsilon^{n+1}+\epsilon^{n}\right)+\frac{1}{2} \delta_{y}^{\beta}\left(\epsilon^{n+1}+\epsilon^{n}\right)+F^{n+1 / 2}+R^{n+1 / 2},
$$

where

$$
\begin{aligned}
F^{n+1 / 2}=\{ & F_{i, j}^{n+1 / 2} \mid F_{i, j}^{n+1 / 2}=f\left(x_{i}, y_{j}, t_{n+\frac{1}{2}}, \frac{3}{2} u_{i, j}^{n}-\frac{1}{2} u_{i, j}^{n-1}, u_{i, j}^{n+\frac{1}{2}-m}\right) \\
& -f\left(x_{i}, y_{j}, t_{n+\frac{1}{2}}, \frac{3}{2} u\left(x_{i}, y_{j}, t_{n}\right)-\frac{1}{2} u\left(x_{i}, y_{j}, t_{n-1}\right), \frac{1}{2} u\left(x_{i}, y_{j}, t_{n+1-m}\right)+u\left(x_{i}, y_{j}, t_{n-m}\right)\right), \\
& \left.1 \leq i \leq M_{x}-1,1 \leq j \leq M_{y}-1\right\}, \quad 0 \leq n \leq N-1 .
\end{aligned}
$$

Furthermore, according to the Lipschitz condition (2.3), we can obtain

$$
\begin{aligned}
& \mid f\left(x_{i}, y_{j}, t_{n+\frac{1}{2}}, \frac{3}{2} u_{i, j}^{n}-\frac{1}{2} u_{i, j}^{n-1}, u_{i, j}^{n+\frac{1}{2}-m}\right) \\
& -f\left(x_{i}, y_{j}, t_{n+\frac{1}{2}}, \frac{3}{2} u\left(x_{i}, y_{j}, t_{n}\right)-\frac{1}{2} u\left(x_{i}, y_{j}, t_{n-1}\right), \frac{1}{2}\left(u\left(x_{i}, y_{j}, t_{n+1-m}\right)+u\left(x_{i}, y_{j}, t_{n-m}\right)\right) \mid\right. \\
\leq & L_{1}\left|\frac{3}{2} \epsilon_{i, j}^{n}-\frac{1}{2} \epsilon_{i, j}^{n-1}\right|+L_{2}\left|\frac{3}{2} \epsilon_{i, j}^{n+1-m}-\frac{1}{2} \epsilon_{i, j}^{n-m}\right| \\
\leq & L_{1}\left(\left|\frac{3}{2} \epsilon_{i, j}^{n}\right|+\left|\frac{1}{2} \epsilon_{i, j}^{n-1}\right|\right)+L_{2}\left(\left|\frac{3}{2} \epsilon_{i, j}^{n+1-m}\right|+\left|\frac{1}{2} \epsilon_{i, j}^{n-m}\right|\right) .
\end{aligned}
$$


Making the inner products of (3.6) with $\epsilon^{n+1 / 2}$, then we have

$$
\begin{aligned}
\left(\delta_{t} \epsilon^{n+1 / 2}, \epsilon^{n+1 / 2}\right)=( & \left.\frac{1}{2} \delta_{x}^{\alpha}\left(\epsilon^{n+1}+\epsilon^{n}\right), \epsilon^{n+1 / 2}\right)+\left(\frac{1}{2} \delta_{y}^{\beta}\left(\epsilon^{n+1}+\epsilon^{n}\right), \epsilon^{n+1 / 2}\right) \\
& +\left(F^{n+1 / 2}, \epsilon^{n+1 / 2}\right)+\left(R^{n+1 / 2}, \epsilon^{n+1 / 2}\right) .
\end{aligned}
$$

Noting that

$$
\begin{aligned}
& \left(\frac{1}{2} \delta_{x}^{\alpha}\left(\epsilon^{n+1}+\epsilon^{n}\right), \epsilon^{n+1 / 2}\right)=\frac{1}{4}\left(\delta_{x}^{\alpha}\left(\epsilon^{n+1}+\epsilon^{n}\right), \epsilon^{n+1}+\epsilon^{n}\right), \\
& \left(\frac{1}{2} \beta_{y}^{\alpha}\left(\epsilon^{n+1}+\epsilon^{n}\right), \epsilon^{n+1 / 2}\right)=\frac{1}{4}\left(\delta_{y}^{\beta}\left(\epsilon^{n+1}+\epsilon^{n}\right), \epsilon^{n+1}+\epsilon^{n}\right),
\end{aligned}
$$

it follows from Lemma 3.3 that

$$
\left(\frac{1}{2} \delta_{x}^{\alpha}\left(\epsilon^{n+1}+\epsilon^{n}\right), \epsilon^{n+1 / 2}\right) \leq 0, \quad\left(\frac{1}{2} \beta_{y}^{\alpha}\left(\epsilon^{n+1}+\epsilon^{n}\right), \epsilon^{n+1 / 2}\right) \leq 0 .
$$

Moreover, we know that

$$
\left(\delta_{t} \epsilon^{n+1 / 2}, \epsilon^{n+1 / 2}\right)=\frac{1}{2 \tau}\left(\epsilon^{n+1}-\epsilon^{n}, \epsilon^{n+1}+\epsilon^{n}\right)=\frac{1}{2 \tau}\left(\left\|\epsilon^{n+1}\right\|^{2}-\left\|\epsilon^{n}\right\|^{2}\right) .
$$

By means of (3.7) and the Cauchy-Schwarz inequality, we have

$$
\begin{aligned}
\left(F^{n+1 / 2}, \epsilon^{n+1 / 2}\right) & \leq\left\|F^{n+1 / 2}\right\| \cdot\left\|\epsilon^{n+1 / 2}\right\| \\
& \leq \frac{1}{2}\left\|L_{1}\left(\left|\frac{3}{2} \epsilon_{i, j}^{n}\right|+\left|\frac{1}{2} \epsilon_{i, j}^{n-1}\right|\right)+L_{2}\left(\left|\frac{3}{2} \epsilon_{i, j}^{n+1-m}\right|+\left|\frac{1}{2} \epsilon_{i, j}^{n-m}\right|\right)\right\| \cdot\left\|\epsilon^{n+1}+\epsilon^{n}\right\| \\
& \leq\left(L_{1}+L_{2}\right)\left(\left\|\epsilon^{n+1}\right\|+\left\|\epsilon^{n}\right\|\right) \cdot\left(\left\|\epsilon^{n}\right\|+\left\|\epsilon^{n-1}\right\|+\left\|\epsilon^{n+1-m}\right\|+\left\|\epsilon^{n-m}\right\|\right) .
\end{aligned}
$$

For the third term on the right side of (3.8), we can obtain

$$
\left(R^{n+1 / 2}, \epsilon^{n+1 / 2}\right) \leq\left\|R^{n+1 / 2}\right\| \cdot\left\|\epsilon^{n+1 / 2}\right\| \leq \frac{1}{2}\left\|R^{n+1 / 2}\right\| \cdot\left(\left\|\epsilon^{n+1}\right\|+\left\|\epsilon^{n}\right\|\right) .
$$

Substituting (3.9)-(3.12) into (3.8), we obtain that

$$
\begin{gathered}
\frac{\left\|\epsilon^{n+1}\right\|^{2}-\left\|\epsilon^{n}\right\|^{2}}{2 \tau} \leq( \\
\left.L_{1}+L_{2}\right)\left(\left\|\epsilon^{n+1}\right\|+\left\|\epsilon^{n}\right\|\right) \cdot\left(\left\|\epsilon^{n}\right\|+\left\|\epsilon^{n-1}\right\|+\left\|\epsilon^{n+1-m}\right\|+\left\|\epsilon^{n-m}\right\|\right) \\
+\frac{1}{2}\left\|R^{n+1 / 2}\right\| \cdot\left(\left\|\epsilon^{n+1}\right\|+\left\|\epsilon^{n}\right\|\right)
\end{gathered}
$$

namely,

$$
\left\|\epsilon^{n+1}\right\| \leq\left\|\epsilon^{n}\right\|+2 \tau\left(L_{1}+L_{2}\right)\left(\left\|\epsilon^{n}\right\|+\left\|\epsilon^{n-1}\right\|+\left\|\epsilon^{n+1-m}\right\|+\left\|\epsilon^{n-m}\right\|\right)+\tau\left\|R^{n+1 / 2}\right\| .
$$


Replacing $n$ by $k$ and summing over $k$ from 1 to $n$, and noticing $\epsilon^{k}=0$ for $-m \leq k \leq 0$, we have

$$
\begin{aligned}
\left\|\epsilon^{n+1}\right\| & \leq\left\|\epsilon^{0}\right\|+8 \tau\left(L_{1}+L_{2}\right) \sum_{k=1}^{n}\left\|\epsilon^{k}\right\|+\tau \sum_{k=1}^{n}\left\|R^{k+1 / 2}\right\| \\
& =8 \tau\left(L_{1}+L_{2}\right) \sum_{k=1}^{n}\left\|\epsilon^{k}\right\|+\tau \sum_{k=1}^{n}\left\|R^{k+1 / 2}\right\| .
\end{aligned}
$$

In view of (3.5), we obtain

$$
\begin{aligned}
\left\|\epsilon^{n+1}\right\| & \leq 8 \tau\left(L_{1}+L_{2}\right) \sum_{k=1}^{n}\left\|\epsilon^{k}\right\|+\tau \sum_{k=1}^{n} c_{1} \sqrt{L_{x} L_{y}}\left(h_{x}^{2}+h_{y}^{2}+\tau^{2}\right) \\
& \leq\left\|\epsilon^{0}\right\|+8 \tau\left(L_{1}+L_{2}\right) \sum_{k=1}^{n}\left\|\epsilon^{k}\right\|+c_{1} T \sqrt{L_{x} L_{y}}\left(h_{x}^{2}+h_{y}^{2}+\tau^{2}\right) .
\end{aligned}
$$

Using the Gronwall Lemma 3.4, we have

$$
\begin{aligned}
\left\|\epsilon^{n+1}\right\| & \leq c_{1} T \sqrt{L_{x} L_{y}} \exp \left(8 n \tau\left(L_{1}+L_{2}\right)\right)\left(h_{x}^{2}+h_{y}^{2}+\tau^{2}\right) \\
& \leq c_{1} T \sqrt{L_{x} L_{y}} \exp \left(8 T\left(L_{1}+L_{2}\right)\right)\left(h_{x}^{2}+h_{y}^{2}+\tau^{2}\right), \quad 0 \leq n \leq N-1 .
\end{aligned}
$$

Consequently,

$$
\left\|\epsilon^{n}\right\| \leq C\left(h_{x}^{2}+h_{y}^{2}+\tau^{2}\right), \quad 1 \leq n \leq N,
$$

where $C=c_{1} T \sqrt{L_{x} L_{y}} \exp \left(8 T\left(L_{1}+L_{2}\right)\right), c_{1}$ is a positive constant satisfies (3.5). The proof is completed.

Next, we will analyze the stability of the scheme (2.15). Let $U_{i, j}^{n}$ be the solution of

$$
\begin{aligned}
& \left(1-\frac{\tau}{2} \delta_{x}^{\alpha}-\frac{\tau}{2} \delta_{y}^{\beta}\right) U_{i, j}^{n+1} \\
= & \left(1+\frac{\tau}{2} \delta_{x}^{\alpha}+\frac{\tau}{2} \delta_{y}^{\beta}\right) U_{i, j}^{n}+\tau f\left(x_{i}, y_{j}, t_{n+\frac{1}{2}}, \frac{3}{2} U_{i, j}^{n}-\frac{1}{2} U_{i, j}^{n-1}, U_{i, j}^{n+\frac{1}{2}-m}\right), \\
& 1 \leq i \leq M_{x}-1, \quad 1 \leq j \leq M_{y}-1 .
\end{aligned}
$$

The boundary and initial conditions are discretized as

$$
\begin{array}{lrl}
U_{0, j}^{n}=u\left(0, y_{j}, t_{n}\right)=0, & U_{M_{x}, j}^{n}=u\left(L_{x}, y_{j}, t_{n}\right)=0, \\
U_{i, 0}^{n}=u\left(x_{i}, 0, t_{n}\right)=0, & U_{i, M_{y}}^{n}=u\left(x_{i}, L_{y}, t_{n}\right)=0, \\
U_{i, j}^{k}=\varphi\left(i h_{x}, j h_{y}, k \tau\right) a+\psi_{i, j}^{k}, & k \leq 0 .
\end{array}
$$

Then, we can obtain the following stability result.

Theorem 3.4. The difference scheme (2.15) is unconditionally stable. 
Proof. Let $u_{i, j}^{n}\left(0 \leq i \leq M_{x}, 0 \leq j \leq M_{y}\right)$ be the numerical solution of (2.15). And $\varepsilon_{i, j}^{n}=U_{i, j}^{n}-u_{i, j}^{n}$, $0 \leq i \leq M_{x}, 0 \leq j \leq M_{y}$ denotes the corresponding error. Then, we have

$$
\begin{aligned}
& \delta_{t} \epsilon_{i, j}^{n+1 / 2}=\frac{1}{2} \delta_{x}^{\alpha}\left(\varepsilon_{i, j}^{n+1}+\varepsilon_{i, j}^{n}\right)+\frac{1}{2} \delta_{y}^{\beta}\left(\varepsilon_{i, j}^{n+1}+\varepsilon_{i, j}^{n}\right)+f\left(x_{i}, y_{j}, t_{n+\frac{1}{2}} \frac{3}{2} U_{i, j}^{n}-\frac{1}{2} U_{i, j}^{n-1}, U_{i, j}^{n+\frac{1}{2}-m}\right) \\
& \quad-f\left(x_{i}, y_{j}, t_{n+\frac{1}{2},} \frac{3}{2} u_{i, j}^{n}-\frac{1}{2} u_{i, j}^{n-1}, u_{i, j}^{n+\frac{1}{2}-m}\right) \\
& \varepsilon_{i, j}^{n}=\psi_{i, j}^{n}, \quad 0 \leq i \leq M_{x}, \quad 0 \leq j \leq M_{y}, \quad-m \leq n \leq 0 \\
& \varepsilon_{i, j}^{n}=0, \quad\left(x_{i}, y_{j}\right) \in \partial \Omega, \quad 1 \leq n \leq N .
\end{aligned}
$$

Similar to the proof of Theorem 3.3, we have

$$
\left\|\varepsilon^{n+1}\right\| \leq\left\|\varepsilon^{0}\right\|+8 \tau\left(L_{1}+L_{2}\right) \sum_{k=1}^{n}\left\|\varepsilon^{k}\right\|+4 \tau L_{2} \sum_{k=-m}^{0}\left\|\varepsilon^{k}\right\| .
$$

It follows from Lemma 3.4 that

$$
\begin{aligned}
\left\|\varepsilon^{n+1}\right\| & \leq\left(\left\|\varepsilon^{0}\right\|+4 \tau L_{2} \sum_{k=-m}^{0}\left\|\varepsilon^{k}\right\|\right) \exp \left(8 n \tau\left(L_{1}+L_{2}\right)\right) \\
& \leq\left(\left\|\varepsilon^{0}\right\|+4 \tau L_{2} \sum_{k=-m}^{0}\left\|\varepsilon^{k}\right\|\right) \exp \left(8 T\left(L_{1}+L_{2}\right)\right) \\
& \leq\left(1+4 s L_{2}\right) \sqrt{L_{x} L_{y}} \exp \left(8 T\left(L_{1}+L_{2}\right)\right)_{\substack{1 \leq i \leq M_{x}-1,1 \leq j \leq M_{y}-1,-m \leq k \leq 0}}\left|\psi_{i, j}^{k}\right| .
\end{aligned}
$$

The proof is completed.

Remark 3.1. Similar to the proofs of Theorem 3.3 and Theorem 3.4, it can be proved that the following results hold

- If the solution of the problem (2.2) satisfy the conditions (A1) and (A2), the method (2.24) is convergent. And the convergence order is $\mathcal{O}\left(h_{x}^{2}+h_{y}^{2}+h_{z}^{2}+\tau^{2}\right)$.

- The difference scheme (2.24) is unconditionally stable.

\section{Numerical examples}

In order to verify and demonstrate our theoretical results, we present some numerical examples in this section. Define the error and convergence orders as

$$
E\left(h_{x}, h_{y}, \tau\right)=\max _{\substack{1 \leq i \leq M_{x}, 1 \leq j \leq M_{y}, 1 \leq n \leq N}}\left|u_{i, j}^{n}-u\left(x_{i}, y_{j}, t_{n}\right)\right|,
$$




$$
\begin{aligned}
& E\left(h_{x}, h_{y}, h_{z}, \tau\right)=\max _{\substack{1 \leq i \leq M_{x}, 1 \leq j \leq M_{y}, 1 \leq k \leq M_{z}, 1 \leq n \leq N}}\left|u_{i, j, k}^{n}-u\left(x_{i}, y_{j}, z_{k}, t_{n}\right)\right| \\
& \text { Order }=\log _{2}\left(\frac{E\left(2 h_{x}, 2 h_{y}, 2 \tau\right)}{E\left(h_{x}, h_{y}, \tau\right)}\right) \quad \text { or } \quad \text { Order }=\log _{2}\left(\frac{E\left(2 h_{x}, 2 h_{y}, 2 h_{z}, 2 \tau\right)}{E\left(h_{x}, h_{y}, h_{z}, \tau\right)}\right) .
\end{aligned}
$$

Example 4.1. Consider the following two-dimensional Riesz space fractional diffusion equations with delay

$$
\left\{\begin{array}{cl}
\frac{\partial u(x, y, t)}{\partial t}= & K_{x} \frac{\partial^{\alpha} u(x, y, t)}{\partial|x|^{\alpha}}+K_{y} \frac{\partial^{\beta} u(x, y, t)}{\partial|y|^{\beta}} \\
\quad+f(x, y, t, u(x, y, t), u(x, y, t-s)), & t \in[0, T], \\
u(x, y, t)=x^{2}(x-1)^{2} y^{2}(y-1)^{2} e^{-t}, & t \in[-s, 0], \quad 0 \leq x \leq 1, \quad 0 \leq y \leq 1, \\
u(0, y, t)=u(1, y, t)=0, & u(x, 0, t)=u(x, 1, t)=0, \quad 0 \leq t \leq T,
\end{array}\right.
$$

where $T=2, s=0.5, K_{x}=K_{y}=1,1<\alpha, \beta \leq 2$,

$$
\begin{aligned}
f( & x, y, t, u(x, y, t), u(x, y, t-s)) \\
=( & u(x, y, t) u(x, y, t-s))^{2}-u(x, y, t)-(x y)^{8}(1-x)^{8}(1-y)^{8} e^{-4 t+2 s} \\
& +\frac{K_{x} e^{-t} y^{2}(y-1)^{2}}{2 \cos \left(\frac{\alpha \pi}{2}\right)}\left\{\frac{24}{\Gamma(5-\alpha)}\left[x^{4-\alpha}+(1-x)^{4-\alpha}\right]\right. \\
& \left.-\frac{12}{\Gamma(4-\alpha)}\left[x^{3-\alpha}+(1-x)^{3-\alpha}\right]+\frac{2}{\Gamma(3-\alpha)}\left[x^{2-\alpha}+(1-x)^{2-\alpha}\right]\right\} \\
& +\frac{K_{y} e^{-t} x^{2}(x-1)^{2}}{2 \cos \left(\frac{\beta \pi}{2}\right)}\left\{\frac{24}{\Gamma(5-\beta)}\left[y^{4-\beta}+(1-y)^{4-\beta}\right]-\frac{12}{\Gamma(4-\beta)}\left[y^{3-\beta}+(1-y)^{3-\beta}\right]\right. \\
& \left.+\frac{2}{\Gamma(3-\beta)}\left[y^{2-\beta}+(1-y)^{2-\beta}\right]\right\},
\end{aligned}
$$

and the exact solution is

$$
u(x, y, t)=x^{2}(1-x)^{2} y^{2}(1-y)^{2} e^{-t} .
$$

One can directly verify that the RHS term satisfies the Lipschitz condition (2.3). When $\alpha=1.6, \beta=1.6$ and $\alpha=1.5, \beta=1.8$, the errors and the computing orders of (2.17)-(2.18) are shown in Table 1 . The numerical results show that the error is very small, and the convergence order is close to 2 . Fig. 1 shows that numerical solutions and absolute errors of (4.1) at $t=1$ for $\alpha=\beta=1.6$ with $h_{x}=h_{y}=\tau=1 / 80$. The corresponding results of (4.1) at $t=1$ with $\alpha=1.6, \beta=1.8$ are shown in Fig. 2. From the above tables and figures, we can see that these numerical results are consistent with the our theoretical results in Section 3.

Example 4.2. Consider the following Riesz space fractional diffusion equations with de- 
Table 1: The errors and convergence orders of the method (2.17)-(2.18).

\begin{tabular}{||ccc|ccc||}
\hline \multicolumn{3}{||c|}{$\alpha=1.6, \beta=1.6$} & \multicolumn{3}{|c|}{$\alpha=1.5, \beta=1.8$} \\
\hline$\tau=h_{x}=h_{y}$ & $E\left(h_{x}, h_{y}, \tau\right)$ & Order & $\tau=h_{x}=h_{y}$ & $E\left(h_{x}, h_{y}, \tau\right)$ & Order \\
$\frac{1}{20}$ & $3.4911 \mathrm{E}-05$ & & $\frac{1}{20}$ & $3.5631 \mathrm{E}-05$ & \\
$\frac{1}{40}$ & $8.5541 \mathrm{E}-06$ & 2.0290 & $\frac{1}{40}$ & $8.7483 \mathrm{E}-06$ & 2.0261 \\
$\frac{1}{80}$ & $2.0944 \mathrm{E}-06$ & 2.0321 & $\frac{1}{80}$ & $2.1493 \mathrm{E}-06$ & 2.0251 \\
$\frac{1}{160}$ & $5.1331 \mathrm{E}-07$ & 2.0286 & $\frac{1}{160}$ & $5.2789 \mathrm{E}-07$ & 2.0256 \\
\hline
\end{tabular}

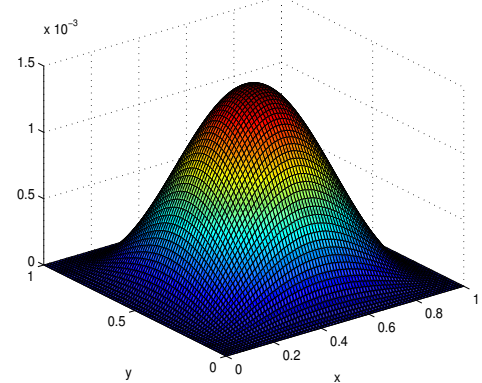

(a)

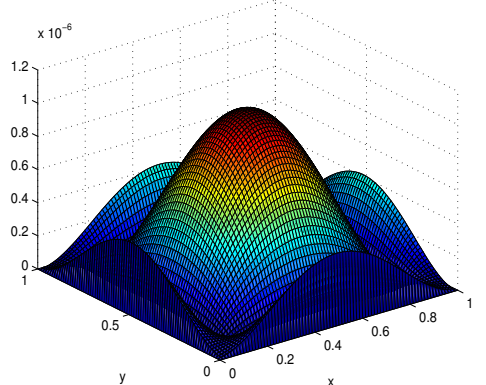

(b)

Figure 1: Numerical solutions and absolute errors of (4.1) by using the method (2.17)-(2.18) at $t=1$ with $\alpha=1.6, \beta=1.6, h_{x}=h_{y}=\tau=1 / 80$, (a) numerical solutions, (b) absolute errors.

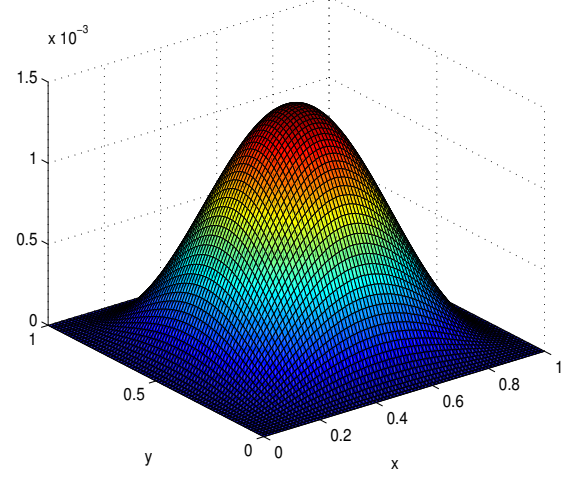

(a)

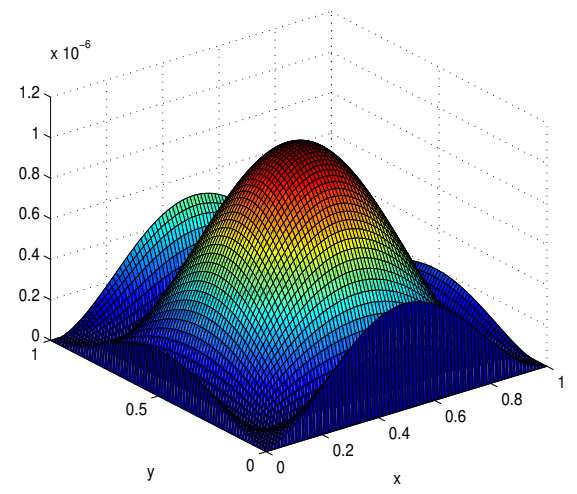

(b)

Figure 2: Numerical solutions and absolute errors of (4.1) by using the method (2.17)-(2.18) at $t=1$ with $\alpha=1.5, \beta=1.8, h_{x}=h_{y}=\tau=1 / 80$, (a) numerical solutions, (b) absolute errors.

lay

$$
\left\{\begin{array}{c}
\frac{\partial u(x, y, z, t)}{\partial t}=K_{x} \frac{\partial^{\alpha} u(x, y, z, t)}{\partial|x|^{\alpha}}+K_{y} \frac{\partial^{\beta} u(x, y, z, t)}{\partial|y|^{\beta}}+K_{z} \frac{\partial^{\gamma} u(x, y, z, t)}{\partial|z|^{\gamma}} \\
\quad+f(x, y, z, t, u(x, y, z, t), u(x, y, z, t-s)), \quad t \in[0, T], \\
u(x, y, z, t)=x^{2}(x-1)^{2} y^{2}(y-1)^{2} z^{2}(z-1)^{2} e^{-t}, \quad t \in[-s, 0] \\
0 \leq x \leq 1, \quad 0 \leq y \leq 1, \quad 0 \leq z \leq 1, \\
u(0, y, z, t)=u(1, y, z, t)=0, \quad u(x, 0, z, t)=u(x, 1, z, t)=0, \\
u(x, y, 0, t)=u(x, y, 1, t)=0, \quad 0 \leq t \leq T,
\end{array}\right.
$$


Table 2: The errors and convergence orders of the method (2.27)-(2.29).

\begin{tabular}{||ccc||}
\hline \multicolumn{4}{|c||}{$\alpha=1.2, \beta=1.2, \gamma=1.2$} \\
\hline$\tau=h_{x}=h_{y}=h_{z}$ & $E\left(h_{x}, h_{y}, h_{z}, \tau\right)$ & Order \\
\hline$\frac{1}{10}$ & $6.3469 \mathrm{E}-06$ \\
$\frac{1}{20}$ & $1.5913 \mathrm{E}-06$ & 1.9958 \\
$\frac{1}{40}$ & $3.9605 \mathrm{E}-07$ & 2.0065 \\
$\frac{1}{80}$ & $9.8278 \mathrm{E}-07$ & 2.0107 \\
\hline \multicolumn{4}{|c||}{$\alpha=1.7, \beta=1.8, \gamma=1.9$} \\
\hline$\tau=h_{x}=h_{y}=h_{z}$ & $E\left(h_{x}, h_{y}, h_{z}, \tau\right)$ & Order \\
\hline$\frac{1}{10}$ & $3.4262 \mathrm{E}-06$ \\
$\frac{1}{20}$ & $1.1281 \mathrm{E}-06$ & 1.6027 \\
$\frac{1}{40}$ & $3.0127 \mathrm{E}-07$ & 1.9048 \\
$\frac{1}{80}$ & $7.4678 \mathrm{E}-08$ & 2.0123 \\
\hline
\end{tabular}

where $T=2, s=0.5, K_{x}=K_{y}=K_{z}=1,1<\alpha, \beta, \gamma \leq 2$,

$$
\begin{aligned}
f( & x, y, z, t, u(x, y, z, t), u(x, y, z, t-s)) \\
=u( & (x, y, z, t) u(x, y, z, t-s)-u(x, y, z, t)-(x y z)^{4}(1-x)^{4}(1-y)^{4}(1-z)^{4} e^{-2 t+s} \\
& +\frac{K_{x} e^{-t} y^{2}(y-1)^{2} z^{2}(z-1)^{2}}{2 \cos \left(\frac{\alpha \pi}{2}\right)}\left\{\frac{24}{\Gamma(5-\alpha)}\left[x^{4-\alpha}+(1-x)^{4-\alpha}\right]\right. \\
& \left.-\frac{12}{\Gamma(4-\alpha)}\left[x^{3-\alpha}+(1-x)^{3-\alpha}\right]+\frac{2}{\Gamma(3-\alpha)}\left[x^{2-\alpha}+(1-x)^{2-\alpha}\right]\right\} \\
& +\frac{K_{y} e^{-t} x^{2}(x-1)^{2} z^{2}(z-1)^{2}}{2 \cos \left(\frac{\beta \pi}{2}\right)}\left\{\frac{24}{\Gamma(5-\beta)}\left[y^{4-\beta}+(1-y)^{4-\beta}\right]\right. \\
& \left.-\frac{12}{\Gamma(4-\beta)}\left[y^{3-\beta}+(1-y)^{3-\beta}\right]+\frac{2}{\Gamma(3-\beta)}\left[y^{2-\beta}+(1-y)^{2-\beta}\right]\right\} \\
& +\frac{K_{z} e^{-t} x^{2}(x-1)^{2} y^{2}(y-1)^{2}}{2 \cos \left(\frac{\gamma \pi}{2}\right)}\left\{\frac{24}{\Gamma(5-\gamma)}\left[z^{4-\gamma}+(1-z)^{4-\gamma}\right]\right. \\
& \left.-\frac{12}{\Gamma(4-\gamma)}\left[z^{3-\gamma}+(1-z)^{3-\gamma}\right]+\frac{2}{\Gamma(3-\gamma)}\left[z^{2-\gamma}+(1-z)^{2-\gamma}\right]\right\},
\end{aligned}
$$

and the exact solution is

$$
u(x, y, z, t)=x^{2}(1-x)^{2} y^{2}(1-y)^{2} z^{2}(1-z)^{2} e^{-t} .
$$

Similarly, one can verify that the RHS term satisfies the Lipschitz condition (2.4). We list the errors and convergence orders of (2.27)-(2.29) in Table 2 with respect to $h_{x}=h_{y}=$ $h_{z}=\tau$. The presented results clearly indicate that the method (2.27)-(2.29) is almost second order accuracy. Fig. 3 shows that the numerical solutions and absolute errors of (4.2) at $t=1, z=0.5$ for $\alpha=\beta=\gamma=1.2$. The corresponding numerical results for (2.27)-(2.29) 


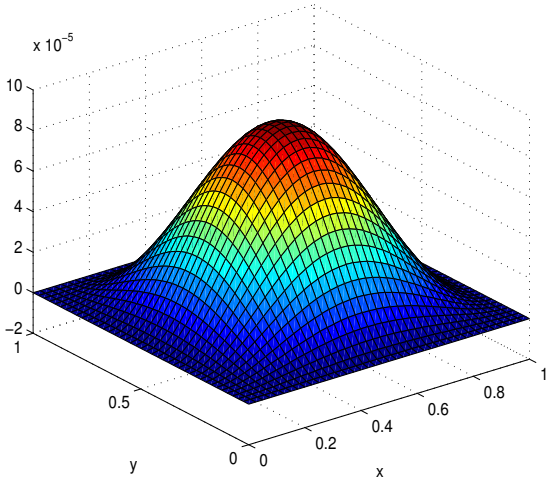

(a)

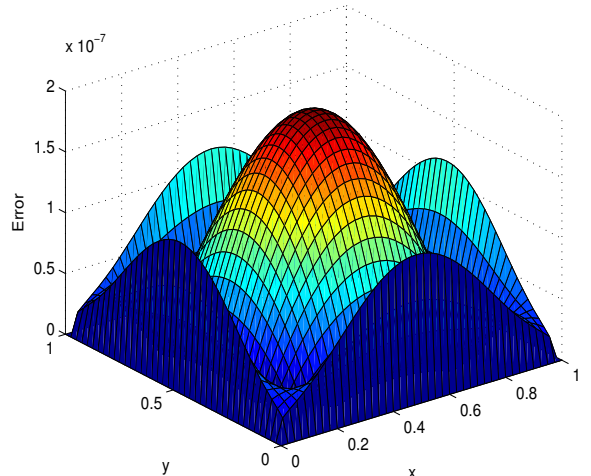

(b)

Figure 3: Numerical solutions and absolute errors of (4.2) by using the method (2.27)-(2.29) at $t=1, z=0.5$ with $\alpha=\beta=\gamma=1.2, h_{x}=h_{y}=h_{z}=\tau=1 / 40$, (a) numerical solutions, (b) absolute errors.

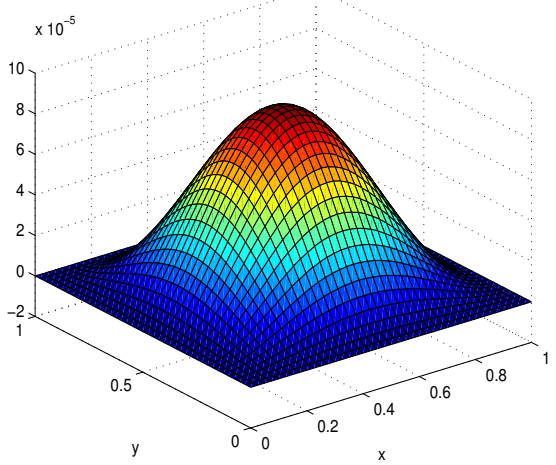

(a)

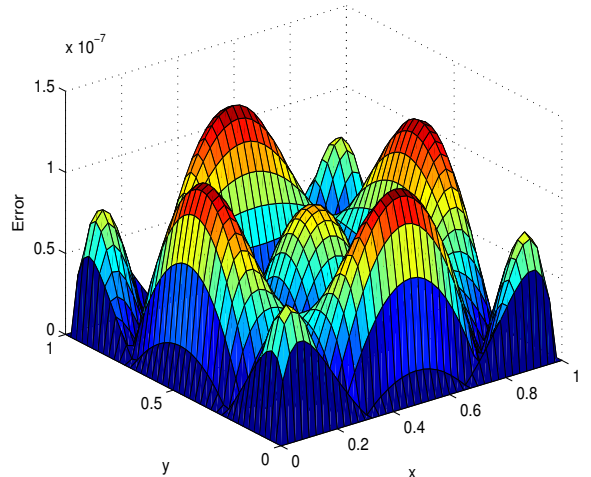

(b)

Figure 4: Numerical solutions and absolute errors of (4.2) by using the method (2.27)-(2.29) at $t=1, z=0.5$ with $\alpha=1.7, \beta=1.8, \gamma=1.9, h_{x}=h_{y}=h_{z}=\tau=1 / 40$, (a) numerical solutions, (b) absolute errors.

with $\alpha=1.7, \beta=1.8, \gamma=1.9$ are shown in Fig. 4 . As we all known, it is very difficult to solve the three-dimensional fractional differential equations. From the above tables and figures, we can see that the proposed methods is efficient and accurate to solve the three-dimensional semilinear Riesz space fractional diffusion equations with time delay.

\section{Conclusions}

In this paper, second order implicit alternating direction methods have been constructed for solving a class of two/three-dimensional semilinear Riesz space fractional diffusion 
equations with time delay subject to homogeneous Dirichlet boundary conditions. We established the sharp stability and convergence estimates for the proposed numerical methods. The benchmarking numerical examples verify the effectiveness and efficiency of our numerical scheme. For the future study, we plan to apply the newly derived numerical methods to some inverse problems in the fractional setting, say in particular the Schiffer problem, which is a longstanding problem in the inverse scattering theory $[4,9,17,18,25,28-38]$, but was recently solved in the fractional setting associated with the fractional Helmholtz equation [5].

\section{Acknowledgements}

The work of S. Yang was partially supported by National Natural Science Foundation (NSF) of China (Grant No. 11501238), NSF of Guangdong Province (Grant No. 2016A030313119) and NSF of Huizhou University (Grant No. hzu201806). The work of H. Liu was supported by the startup fund from City University of Hong Kong and the Hong Kong RGC General Research Fund (projects Nos. 12301420, 12302919 and 12301218). The work of C. Wang was partially supported by the NSF of China No. 11971221, the Shenzhen Sci-Tech Fund No. JCYJ20190809150413261, JCYJ201803071516 03959, and JCYJ20170818153840322, and Guangdong Provincial Key Laboratory of Computational Science and Material Design (No. 2019B030301001).

\section{References}

[1] A. Ashyralyev, And D. Agirseven, On convergence of difference schemes for delay parabolic equations, Comput. Math. Appl., 66 (2013), pp. 1232-1244.

[2] S. Bhalekar, V. DAftardar-GejJi, D. BAleAnU, AND R. MAGin, Fractional Bloch equation with delay, Comput. Math. Appl., 61 (2011), pp. 1355-1365.

[3] S. BHALEKAR AND V. DAFTARDAR-GEJJI, A predictor-corrector scheme for solving nonlinear delay differential equations of fractional order, J. Fractional Calculus Appl., 1 (2011), pp. 1-9.

[4] X. CAO, H. DIAO, H. LIU AND J. ZOU, On nodal and singular structures of Laplacian eigenfunctions and applications to inverse scattering problems, Journal de Mathématiques Pures et Appliquées, 143 (2020), pp. 116-161.

[5] X. CAO, Y.-H. LIN AND H. LIU, Simultaneously recovering potentials and embedded obstacles for anisotropic fractional Schrodinger operators, Inverse Problems Imag., 13 (2019), pp. 197-210.

[6] X. CAO AND H. LIU, Determining a fractional Helmholtz system with unknown source and medium parameter, Commun. Math. Sci., 17(7) (2019), pp. 1861-1876.

[7] S. CHEN, F. LiU, X. JiAng, I. TURner AND V. ANH, A fast semi-implicit difference method for a nonlinear two-sided space-fractional diffusion equation with variable diffusivity coefficients, Appl. Math. Comput., 257 (2015), pp. 591-601.

[8] V. DAFTARDAR-GejJi, Y. SuKAlE, AND S. BHAlEKAR, Solving fractional delay differential equations: A new approach, Fractional Calculus Appl. Anal., 18 (2015), pp. 400-418. 
[9] H. DiAO, H. LiU AND L. WANG, On generalized Holmgren's principle to the Lamé operator with applications to inverse elastic problems, Calculus of Variations and Partial Differential Equations, 59(5) (2020), 179.

[10] K. DieTHELM, An algorithm for the numerical solution of differential equations of fractional order, Electronic Trans. Numer. Anal., 5 (1997), pp. 1-6.

[11] K. Diethelm, AND G. WAlz, Numerical solution of fractional order differential equations by extrapolation, Numer. Algorithms, 16 (1997), pp. 231-253.

[12] K. DieTHELM, N. J. FORD AND AlAN D. FREED, A predictor-corrector approach for the numerical solution of fractional differential equations, Nonlinear Dyn., 29 (2002), pp. 3-22.

[13] Z. DING, A. XIAO, AND M. LI, Weighted finite difference methods for a class of space fractional partial differential equations with variable coefficients, J. Comput. Appl. Math., 233(8) (2010), pp. 1905-1914.

[14] L. B. FenG, FAWANG LiU, I. TURner, Q. Q. YANG, AND P. H. ZHUANG, Unstructured mesh finite difference/finite element method for the $2 D$ time-space Riesz fractional diffusion equation on irregular convex domains, Appl. Math. Model., 59 (2018), pp. 441-463.

[15] Z. HAO, K. FAN, W. CAO, AND Z. SUN, A finite difference scheme for semilinear space-fractional diffusion equations with time delay, Appl. Math. Comput., 275 (2016), pp. 238-254.

[16] C. M. HuAnG, AND S. VANDEWALle, Analysis of delay-dependent stability for ordinary and partial differential equations with fixed and distributed delays, SIAM J. Sci. Comput., 25 (2004), pp. $1608-1632$.

[17] J. LI, H. LIU, Z. SHANG AND H. SUN, Two single-shot methods for locating multiple electromagnetic scatterers, SIAM J. Appl. Math., 73 (2013), pp. 1721-1746.

[18] J. LI, H. LIU AND Q. WANG, Locating multiple multi-scale electromagnetic scatterers by a single far-field measurement, SIAM J. Imag. Sci., 6 (2013), pp. 2285-2309.

[19] F. LiU, P. ZhUANG, V. ANH, I. TURner AND K. Burrage, Stability and convergence of the difference methods for the space-time fractional advection-diffusion equation, Appl. Math. Comput., 191 (2007), pp. 12-20.

[20] F. LiU, P. H. ZhuANG, AND Q. X. LiU, Numerical Methods of Fractional Partial Differential Equations and Their Applications, Beijing Science Press (in Chinese), 2015.

[21] F. LiU, S. Chen, AND I. TURNeR ET AL., Numerical simulation for two-dimensional Riesz space fractional diffusion equations with a nonlinear reaction term, Central Euro. J. Phys., 11(10) (2013), pp. 1221-1232.

[22] F. Liu, P. Zhuang, I. Turner, K. Burrage And V. Anh, A new fractional finite volume method for solving the fractional diffusion equation, Appl. Math. Model., 38(15-16) (2014), pp. 3871-3878.

[23] H. LIANG, Convergence and asymptotic stability of Galerkin methods for linear parabolic equations with delays, Appl. Math. Comput., 264 (2015), pp. 160-178.

[24] H. LIANG, M. Z. LIU, AND W. J. LV, Stability of $\theta$-schemes in the numerical solution of a partial differential equation with piecewise continuous arguments, Appl. Math. Lett., 23 (2010), pp. 198206.

[25] J. Li AND H. LIU, Recovering a polyhedral obstacle by a few backscattering measurements, Journal of Differential Equations, 259 (2015), pp. 2101-2120.

[26] M. Li, J. Li, R. MARTIN AND K. ZHANG, A numerical framework to simplify CAD models for reliable estimates of physical quantities, Adv. Appl. Math. Mech., 11 (2019), pp. 870-889.

[27] M. LI, L. ZHU, J. LI AND K. ZHANG, Design optimization of interconnected porous structures using extended triply periodic minimal surfaces, J. Comput. Phys., 425 (2021), pp. 109909.

[28] H. LIU, A global uniqueness for formally determined inverse electromagnetic obstacle scattering, 
Inverse Problems, 24 (2008), 035018.

[29] H. LIU, On recovering polyhedral scatterers with acoustic far-field measurements, IMA J. Appl. Math., 74 (2009), pp. 264-272.

[30] H. LiU, M. PETRINI, L. RONDi AND J. XIAO, Stable determination of sound-hard polyhedral scatterers by a minimal number of scattering measurements, Journal of Differential Equations, 262(3) (2017), pp. 1631-1670.

[31] H. LIU, L. RONDI AND J. XIAO, Mosco convergence for H(curl) spaces, higher integrability for Maxwell's equations, and stability in direct and inverse EM scattering problems, Journal of the European Mathematical Society (JEMS), 21(10) (2019), pp. 2945-2993.

[32] H. LIU AND J. XIAO, Decoupling elastic waves and its applications, Journal of Differential Equations, 265(8) (2017), pp. 4442-4480.

[33] H. LIU, M. YAMAMOTO AND J. ZOU, Reflection principle for Maxwell's equations and its application to inverse electromagnetic scattering problem, Inverse Problems, 23 (2007), pp. 2357-2366.

[34] H. LIU AND J. ZOU, Uniqueness in an inverse acoustic obstacle scattering problem for both soundhard and sound-soft polyhedral scatterers, Inverse Problems, 22 (2006), pp. 515-524.

[35] H. LIU AND J. ZOU, On unique determination of partially coated polyhedral scatterers with farfield measurements, Inverse Problems, 23 (2007), pp. 297-308.

[36] H. LIU AND J. ZOU, Zeros of Bessel and spherical Bessel functions and their applications for uniqueness in inverse acoustic obstacle scattering problems, IMA J. Appl. Math., 72 (2007), pp. 817-831.

[37] H. LIU, AND J. ZOU, Uniqueness in determining multiple polygonal scatterers of mixed type, Discrete and Continuous Dynamical Systems, Series B, 9(2) (2008), pp. 375-396.

[38] H. LIU AND J. ZOU, On uniqueness in inverse acoustic and electromagnetic obstacle scattering problems, J. Phys. Conference Series, 124 (2008), 012006.

[39] R. L. MAGIN, Fractional calculus in bioengineering, Critical Reviews in Biomedical Engineering, 32(1) (2004), pp. 1-104.

[40] Z. OUYANG, Existence and uniqueness of the solutions for a class of nonlinear fractional order partial differential equations with delay, Comput. Math. Appl., 61 (2011), pp. 860-870.

[41] P. PRAKASH, S. CHOUdHARY, AND V. DAFTARDAR-GEJJI, Exact solutions of generalized nonlinear time-fractional reaction-diffusion equations with time delay, Euro. Phys. J. Plus, 135 (2020), 490. https://doi.org/10.1140/epjp/s13360-020-00445-1.

[42] I. Podlubny, Fractional Differential Equations, Academic Press, New York, 1999.

[43] I. PETRÁš, Modeling and numerical analysis of fractional-order Bloch equations, Comput. Math. Appl., 61 (2011), pp. 341-356.

[44] A. QuARteroni, R. SACCO, AND F. SAleri, Numerical Mathematics, Springer-Verlag, 2007.

[45] M. RAN, AND Y. HE, Linearized Crank-Nicolson method for solving the nonlinear fractional diffusion equation with multi-delay, Int. J. Comput. Math., 95(12) (2018), pp. 2458-2470.

[46] G. D. SIMITH, Numerical Partial Differential Equations: Finite Difference Methods, Oxford: Oxford University Press, 1978.

[47] I. M. SOKOlOV, J. Klafter, AND A. Blumen, Fractional kinetics, Phys. Today, 55(11) (2002), pp. $48-54$.

[48] H. H. SUN, B. ONARAL, AND Y. TSAO, Application of positive reality principle to metal electrode linear polarization phenomena, IEEE Transactions on Biomedical Engineering, BME-31(10) (1984), pp. 664-674.

[49] H. H. Sun, A. A. ABDelwahAB, AND B. ONARAL, Linear approximation of transfer function with a pole of fractional order, IEEE Transactions on Automatic Control, AC-29(5) (1984), pp. 
$441-444$.

[50] Z. Sun, AND G. H. GAO, The Finite Difference Method for Fractional Differential Equations, Beijing Science Press, (in Chinese), 2015.

[51] D. WANG, A. XIAO, AND W. YANG, Crank-Nicolson difference scheme for the coupled nonlinear Schrödinger equations with the Riesz space fractional derivative, J. Comput. Phys., 242(242) (2013), pp. 670-681.

[52] M. ZAYERNOURI, W. CAO, Z. ZHANG, AND G. E. KARNIADAKIS, Spectral and discontinuous spectral element methods for fractional delay equations, SIAM J. Sci. Comput., 36 (2014), pp. B904B929.

[53] F. ZENG, AND F. LIU ET AL., A Crank-Nicolson adi spectral method for a two-dimensional riesz space fractional nonlinear reaction-diffusion equation, SIAM J. Numer. Anal., 52(6) (2014), pp. 2599-2622.

[54] D. ZHANG, Y. GUO, J. LI AND H. LIU, Locating multiple multipolar acoustic sources using the direct sampling method, Commun. Comput. Phys., 25 (2019), pp. 1328-1356.

[55] J. ZHANG, K. ZHANG, J. LI AND X. WANG, A weak Galerkin finite element method for the Navier-Stokes equations, Commun. Comput. Phys., 23 (2018), pp. 706-746.

[56] Y. ZHANG, AND H. DING, High-order algorithm for the two-dimension Riesz space-fractional diffusion equation, Int. J. Comput. Math., 94(10) (2017), pp. 2063-2073.

[57] X. ZHAO, Z. SUN, AND G. E. KARNIADAKIS, Second-order approximations for variable order fractional derivatives: Algorithms and applications, J. Comput. Phys., 293 (2015), pp. 184-200.

[58] X. ZHU, Y. NIE, AND J. WANG ET AL., A numerical approach for the Riesz space-fractional Fisher's equation in two-dimensions, Int. J. Comput. Math., 94(2) (2017), pp. 296-315.

[59] P. ZHUANG, F. LIU, AND V. ANH ET AL. Numerical methods for the variable-order fractional advection-diffusion equation with a nonlinear reaction term, SIAM J. Numer. Anal., 47(3) (2009), pp. 1760-1781.

[60] M. ZHENG, F. LIU, I. TURNER AND V. ANH, A novel high order space-time spectral method for the time-fractional Fokker-Planck equation, SIAM J. Sci. Comput., 37(2) (2015), pp. A701-A724.

[61] F. ZENG, F. LiU, C. LI, K. BurRAGE, I. TURNER AND V. ANH, Crank-Nicolson ADI spectral method for the two-dimensional Riesz space fractional nonlinear reaction-diffusion equation, SIAM J. Numer. Anal., 52-6 (2014), pp. 2599-2622.

[62] F. ZENG, C. LI, F. LIU AND I. TURNER, The use of finite difference/element approximations for solving the time-fractional subdiffusion equation, SIAM J. Sci. Comput., 35(6) (2013), pp. 29763000 .

[63] H. WANG, Y. CHEN, Y. HuANG, AND W. MAO, A posteriori error estimates of the Galerkin spectral methods for space-time fractional diffusion equations, Adv. Appl. Math. Mech., 12(1) (2020), pp. 87-100.

[64] Y. YANG, J. TAO, S. ZHANG, AND P. V. SIVTSEV, A Jacobi collocation method for the fractional Ginzburg-Landau differential equation, Adv. Appl. Math. Mech., 12(1) (2020), pp. 57-86. 